\title{
PCA based Optimization using Conjugate Gradient Descent Algorithm
}

\author{
Subhas A. Meti \\ Department of Electronics and Communication Engineering, \\ Research Scholar, VTU Regional Research Center, \\ Belgaum, India
}

\author{
V.G. Sangam \\ Department of Electronics and Instrumentation, \\ Dayanand Sagar College of Engineering, \\ Bangalore, India
}

\begin{abstract}
The energy dissipation in Wireless Body Area Network (WBAN) systems is the biggest concern as it proportionally affects the system longevity. This energy dissipation in the WBAN system mainly takes place due to the signal interference from other networks causing reduction on the dimensionality. The data prediction in WBAN is also a considerable concern corresponding to misinterpretations and faults in the signals. In this paper a novel combination of Principle Component Analysis (PCA) pre-processing along with optimization using the conjugate gradient descent algorithm is proposed. Experimental observations show an improvement in the mean square error and the regression based correlation coefficient when compared to other standard techniques.
\end{abstract}

Keywords-Associative neural network (AANN); conjugate gradient descent; Non-Linear Principle Component Analysis (NLPCA); Principle Component Analysis (PCA); Wireless Body Area Network (WBAN)

\section{INTRODUCTION}

The effective improvement in the wireless communication area corresponding to the wireless sensor network (WSN) providing the wide range applications in different areas like military, medical, etc. A kind of WSN is named as Wireless Body Area Network (WBAN) helps to connect the different medical sensor within and outside the body. These WBANs offers the significant mobility for the patients by portable monitoring gadgets. The monitoring ability of the WBAN is area independent and can access the data network to transfer the patient's data. Thus, WBAN framework likewise could get to the information systems (e.g. 3G, 4G) to transfer the patients data. The prime concern of WBAN framework is the productivity regarding energy which demonstrates the system lifetime. The energy in the WBAN could be influenced by numerous variables in light of the area of the observing gadgets which produces clamor/obstruction in the sign. The conventional rarities created from other similar gadgets could be because of variables, for example, state of the checking gadgets, interference from other medicinal sensors, and so on. The conventional methods delivered from inside restorative gadgets incorporate impedance of different signs created because of inadvertent physiological criteria. Subsequently, the restorative gadgets of WBAN system produces signal where antiquities may exist. This kind of interference misjudges the sign that injects the errors and along these lines prompting inappropriate signal forecast. The use of neural networks for WBAN systems helps to enhance the system efficiency, signal prediction and artifact reduction. In order to describe the problem of energy dissipation in WBAN, An enhancement was done on the WBAN framework in view of the arrangement of the facilitator utilizing neural system strategies [1]. A learning algorithm was used with Kohonen neural system (KNN) to analyze and classify the biomedical signals in the WBAN framework [2]. By utilizing learning based techniques as a part of the neural systems the general energy utilization was lessened to $90 \%$. One of the issues tended to in the WBAN systems is the planning of numerous WBAN in a specific region. The work of [3] considered the same issue of nonlinearity to achieve the system high throughput. If a WBAN system exists in a system of multiple WBAN then dimensionality issue may take place, due to which the communication performance may vary because of channel interface among the WBAN systems [4]. Thus, there is a need of a method which can reduce the dimensions in multiple WBAN systems. The analysis and fault detection of biomedical sensors can be done through the modular neural network consisting of associative neural network (AANN). The significant feature of AANN is that it interprets the obtained outcomes and it can be analyzed through data spaces correlation in space modes, which adversely helps in improvement of prediction capability in WBAN system.

This paper is planned as per the sections, where Section II represents the existing research work highlighting the advantage of learning based AANN algorithm. The Section III explains the problem of interest while the next Section IV explains the general modules used in the proposed system. The Section $\mathrm{V}$ is subjected to describe the research methodology of the proposed model. The Section VI illustrates the analysis of the outcomes of the system. Finally the Section VII briefs about the conclusion of the proposed system.

\section{RELATED WORK}

In past various learning based mechanisms were introduced for different application needs. The AANN is a method which falls under the associated neural network (ASNN). This section discussed few existing researches pertaining to ASNN.

The work of Guo-Jian et al. [5] expressed a self-restoration mechanism for the intelligent sensors that implements the AANN to monitor the online insulator contamination status by performing learning. The outcomes of the study suggested that 
the use of the AANN based learning based mechanism can locate two faulty transducers synchronously and also the method was able to estimate and recover the drift failures in $<5$ seconds.

In combined work of Gupta and Singh [6] a bidirectional associative memory (BAM) $\mathrm{NN}$ is used to develop a string based NN system for recognition. The study lags with the storage and retrieval of pattern issue as per the BAM efficiency is concerned.

The method of Ang et al. [7] uses a Spiking neural network method to interconnect the delays through FPGA implementation. This study adopted the AANN based memory structure. The outcomes suggests that the method was able to perform the data recalling and learning up to four input pattern by implementing the temporal coding. The obtained neuron response was reduced about 1 to $2 \mathrm{~ms}$ by reconfiguring the $60 \mathrm{~ns}$ pulse width.

The collaborative work of Lemma and Hasim [8] explained the fault recognition using gas turbine recognition system by introducing a combination model of both AANN and wavelet transformation. The outcomes of the study found bias of $\sim 10 \%$ with average detection rate of $95 \%$.

The work of Ravi et al. [9] considered an ANN based banking application where training and classification methods are used for bankruptcy prediction datasets. The implemented AANA method in this study was an optimized method and is named as "Bio-inspired swarm intelligent" model with ASNN. The component analysis was done to reduce the data dimensionality. This [9] study has acquired the higher accuracy than the previous methods.

The study introduced by Gerimella et al. [10] gives the Regularized AANN model based "speaker verification model". Here the noise generates due to the outliers are considered for regularized AANN model and the parameters of this models are used for Speaker verification model which implements the Linear Discriminate Analysis (LDA). The final outcomes suggested that the EER is improved.

The significant work of Chakraborty [11] introduces a Cardiac Arrhythmia based classification model by using the AANN method. The classifier used in this extracts the nonrequired features and the study outcome with the high accuracy of $>97 \%$ and relative gain of $>90 \%$.

In Krstulovic et al. [12] a local AANN based power estimation model was introduced. In this Kirchoff's low is considered as solution for topology constrains and helps to form the specific pattern. This study suggests that under topology constraints the AANN based system is more useful.

The work of Zin et al. [13] gives a multidimensional data visualization and compression system by considering ANN to interpret the multidimensional data. The final outcomes of [13] research gives that the multidimensional data can only be compressed to few non-linear principle components.

In Ito et al. [14] have described a AANN based face recognition system to generate a learning scheme for various components representing facial variation of expressions. The study found accuracy of $77 \%$.
The work addressed in Wang and Yanying [15] states the biometric system for appearance based face recognition. In this, the AANN is considered over one person. The research outcome with the increased recognition rate under partial occlusion and nose of image.

A work addressed in Zhang and Zhou [16] represents a face recognition system for video data by using AANN, in which two facial images were compared for recognition. The accuracy of $90 \%$ was obtained under temporal aspects of face image during its recognition.

In the next section, the identified problem is illustrated.

\section{PROBLEM IDENTIFICATION}

This paper addresses multiple issues in the domain of WBAN. The energy dissipation in the WBAN exhibits factors like dimensionality, signal prediction, cross interference, computational time, etc. All these factors contribute to the energy dissipation which in turn leads to reduced network lifetime. Some of the issues addressed in this paper are as follows:

- Reduction of dimensionality: The interference of multiple WBAN in some areas. The dimensionality issue signifies the pervasive in this scenario, there is a necessity of dimensionality mapping (from higher dimensional to lower dimensional subspace. In such case the data may be of non-linear and hence data decomposition is need to be performed or dimensionality reduction.

- Gradient Descent in node training: For nonlinear data to reduce the dimensionality the Multi Perception Training (MLP) can be implemented. The MLP overhauls just the weights and totally overlooks the status of the inputs. This makes the framework to consider additionally preparing tests which makes the framework redundant. Thus there is a prerequisite to consider upgrading weights alongside its inputs. For this reason a Non-Linear Principle Component Analysis (NLPCA) is performed. In the NLPCA both the weights and the inputs are overhauled unlike other MLP techniques.

- Machine learning application to WBAN: The mapping methods implemented for feature extraction is a preparatory step with regards to machine learning. The following are the important stage in the proposed recognition system which is acknowledged in the mapped data. This requires the utilization of pattern recognition calculations. The example pattern recognition techniques ranges from unsupervised learning mechanisms i.e., Multi-linear Principal Component Analysis (MPCA) to supervised learning (regression based) methods, and for linear regression, Gaussian process regression, neural networks and Deep learning techniques.

- Non-Linear Optimization in WBAN: The problem associated with non-linear optimization which is difficult to provide optimal solution given the objective function as to maximize throughput. The work in $\mathrm{Li}$ et 
al. [1] details this problem by introducing a system called "Fairness-based Throughput Maximization Heuristic (FTMH)" which gives "suboptimal solution" with reduced complexity. Considering the WBAN system of linear equations, enhancement could likewise be performed using techniques, like Conjugate Gradient Algorithms.

\section{PROPOSED SYSTEM}

This gives the modules used in the proposed system to tackle the energy dissipation issue. Fig. 1 represents the block diagram of proposed system.

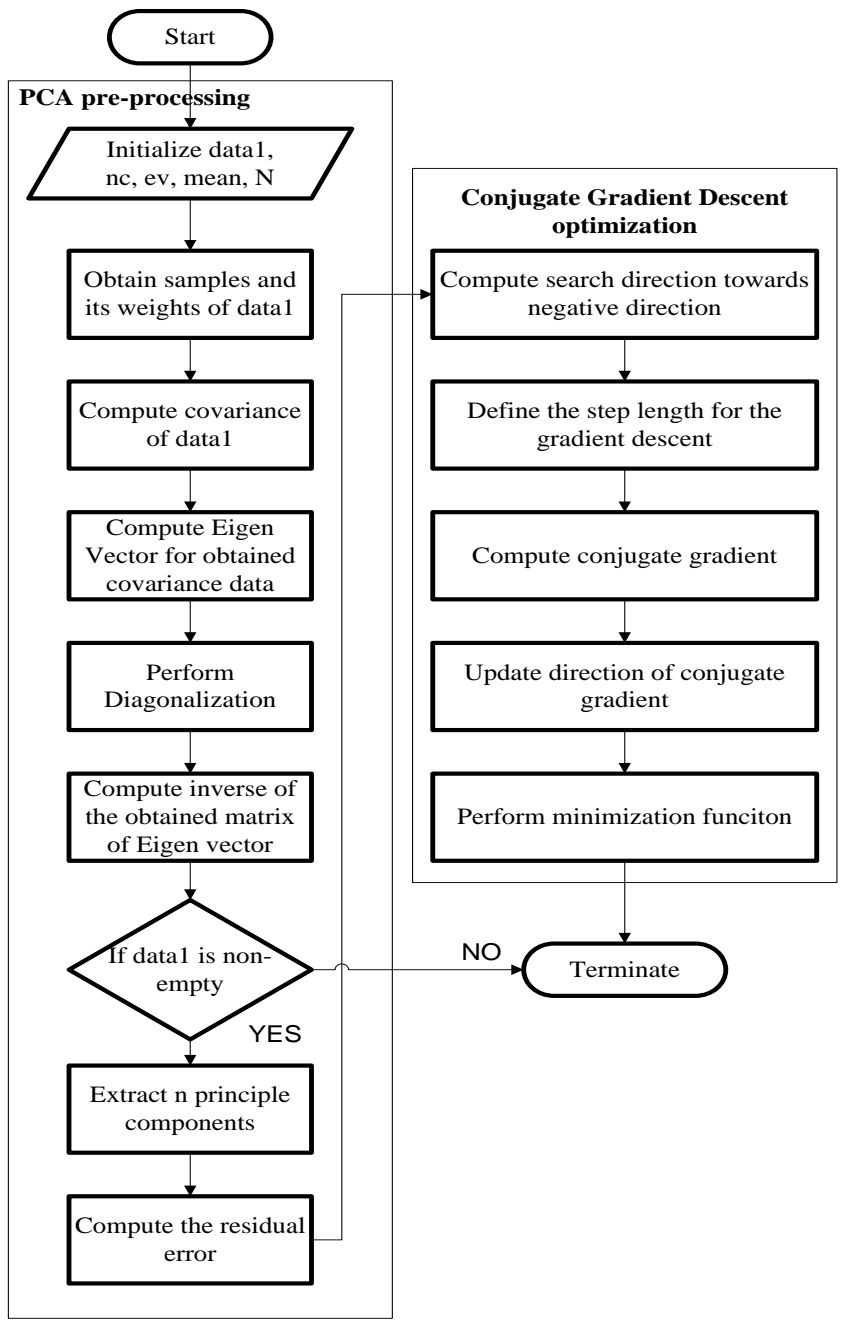

Fig. 1. Proposed system.

- The system contains the preprocessing stage which performs the PCA extraction. In the system, the component analysis (nonlinear principle) method is used for dimensionality reduction.

- The next stage consists of optimization method based on conjugate gradient. In this the supervised (regression) learning mechanism of ANN implemented. Also a modular (based on associative memory) learning mechanism of ANN implemented.
- For training phase AANN method is used which automatically generates the desired training samples for prediction.

\section{RESEARCH METHODOLOGY}

A WBAN system is mainly composed of more medical devices interconnection which forms a network. Some of the challenges associated with WBAN system are highlighted in Table 1. Practically, the application of WBAN associated with medical devices used for inner body (internal) gives the issues occurred due to signal interference (from same devices) or unfamiliar physical behavior. The electroencephalogram (EEG) is a signal which measures the brain activities by using electrical signals generated by brains where noise may be generated (unfamiliar signal interference). The similar thing can be found in electrocardiography (ECG) which helps in heart rate measurement.

The main cause of energy dissipation in the WBAN system is signal interference from system to system of WBAN, WBAN scheduling, WBAN fault detection, WBAN analysis, etc. Hence the energy dissipation is more crucial factor in WBAN system as it impacts the longevity of network directly. In recent years various techniques were introduced to tackle the energy dissipation issue. The scheduling of WBAN is indicated as optimization problem and is non-linear in nature where obtaining the maximum throughput is main objective [1]. Also, the reduction of dimension in WBAN is indicated with the techniques like component reduction and extraction performed by component analysis mechanism. The modular NN based associative memory is presented to detect the fault and analyze the WBAN system.

Also, with all the addressed above problems of WBAN, to make the capable system for real time applications computational efficiency need to be considered. This computational efficiency is mainly has impact on data training and prediction for WBAN system.

In this section, the implementation of the system is discussed. First the data for training is considered which is represented as data1 which consists of samples along its columns and its respective weights along its rows. Data1 is sent for the preprocessing stage which extracts principle components and is further performed an optimization with respect to conjugate gradient descent algorithm. The following section explains steps involved in the process in detail:

- Pre-processing of Principle Component Analysis: Prior to performing the computation for the gradient descent algorithm. The considered data is first applied a preprocessing module whose primary function is to extract the first $\mathrm{m}$ number of principle components using Algorithm 1. The data considered in this process consists of samples which are represented along the columns ( $\mathrm{x}$ ) and their respective weights along its rows (y). This data is represented as data 1.

The covariance is calculated with respect to $\mathrm{x}$ and $\mathrm{y}$ of data 1 , which is represented as shown in (1):

$$
\operatorname{cov}(x, y)=E[(X-E[X])(Y-E[Y])]
$$


Where, $\mathrm{X}, \mathrm{Y} \rightarrow$ rows and columns of data, respectively

$\mathrm{E}[\mathrm{x}], \mathrm{E}[\mathrm{y}] \rightarrow$ mean of $\mathrm{X}$ and $\mathrm{Y}$, respectively

Following the computation of the covariance of the matrix, the Eigen vectors for the obtained covariance is computed which is given in (2):

$$
T(v)=\lambda(\operatorname{cov}(x, y))
$$

Where, $\mathrm{v} \rightarrow$ Eigen vector

\section{$\lambda \rightarrow$ Eigen value}

Equation (2) is alternatively represented as given in (3),

$$
(T-\lambda I) v=0
$$

Where, I $\rightarrow$ Identity matrix

$\mathrm{T} \rightarrow$ transformation matrix

Considering the obtained Eigen vectors which are linearly independent, A square matrix which consists of $\mathrm{n}$ linearly independent vectors is defined which is given in (4) as shown below:

$$
Q=\left[\begin{array}{lll}
v_{1} v_{2} & \ldots & v_{n}
\end{array}\right]
$$

Where, $\mathrm{Q} \rightarrow$ square matrix consisting of $\mathrm{n}$ linear independent Eigen vectors

$$
v_{1} v_{2} \ldots v_{n} \rightarrow \text { Eigen vectors }
$$

The matrix containing the linearly independent Eigen vectors is multiplied with their corresponding Eigen value which is given in (5) as shown below:

$$
A Q=\left[\lambda_{1} v_{1} \lambda_{2} v_{2} \ldots \lambda_{n} v_{n}\right]
$$

The diagonal elements along the matrix AQ are obtained which considers the $i^{\text {th }}$ column of the matrix defined in (4). The same is represented in (6) as follows:

$$
A Q=Q \Lambda
$$

Where, $\Lambda \rightarrow$ diagonal matrix consisting of Eigen value associated with ith column of $\mathrm{Q}$.

The matrix $\mathrm{Q}$ is invertible due the consideration that the columns present in the matrix in (5) is linearly independent. Hence, by multiplying $Q^{-1}$ on both sides, we get,

$$
A=Q \Lambda Q^{-1}
$$

TABLE I. CHALLENGES OF WBAN SYSTEMS

\begin{tabular}{|l|l|l|}
\hline Sl. No & Issues & Energy dissipation \\
\hline 1 & Multiple WBAN scheduling & \\
\hline 3 & $\begin{array}{l}\text { Inference between multiple WBAN systems } \\
\text { (Dimensionality issue) }\end{array}$ & \\
\hline 4 & Fault detection and analysis & \\
\hline
\end{tabular}

The above (7) represents the Eigen decomposition which is considered from a similarity transform. The $\mathrm{n}$ principle components are then extracted using the following equation:

$$
p c a_{\text {data }}=v \times\left(\text { data } 1-\sum_{i=1}^{N}\left[d_{\text {mean }}(i)\right]\right)
$$

Where, $p c a_{\text {data }} \rightarrow$ principle components

$$
d_{\text {mean }} \rightarrow \text { Mean of the principle components }
$$

The residual error post subsequent extraction of the principle components are given as shown in (7).

\begin{tabular}{ll}
\hline Methods Applied \\
- & Learning based algorithm [1] \\
- & Efficient design of routing protocols \\
- & Non-linear optimization with an objective norm of maximum \\
& throughput [1]. \\
- & Clique base WBAN scheduling [17] \\
- & Coloring based scheduling [18] \\
- & Intra WBAN based scheduling [18] \\
- & Component extraction, component identification and analysis such as \\
- & ChA. \\
- & Design of WBANel estimation for efficient dimensionality reduction [19] \\
- & Modular neural network based associative memory for training and \\
& prediction. \\
- & SVM based models [20] \\
Linear regression models [20]
\end{tabular}

$$
\text { res }_{\text {error }}=\sum \sum_{i=1}^{N}{\frac{\left(v \times\left(\text { data } 1-\sum_{i=1}^{N}\left[d_{\text {mean }}(i)\right]\right)^{2}\right.}{d_{-} \text {org }}}^{2}
$$

Where, reserror $\rightarrow$ residual error post extraction of principle components.

The next stage in the proposed system is the learning stage where the respective machine learning algorithm is employed. Optimization is to be performed, in this case a particular type of optimization called as the convex optimization is employed which is possible to the assumption that the system concerning the WBAN is a system of linear equations. The optimization algorithm used in this case is the Conjugate Gradient Descent based optimization as stated in Algorithm 2. 


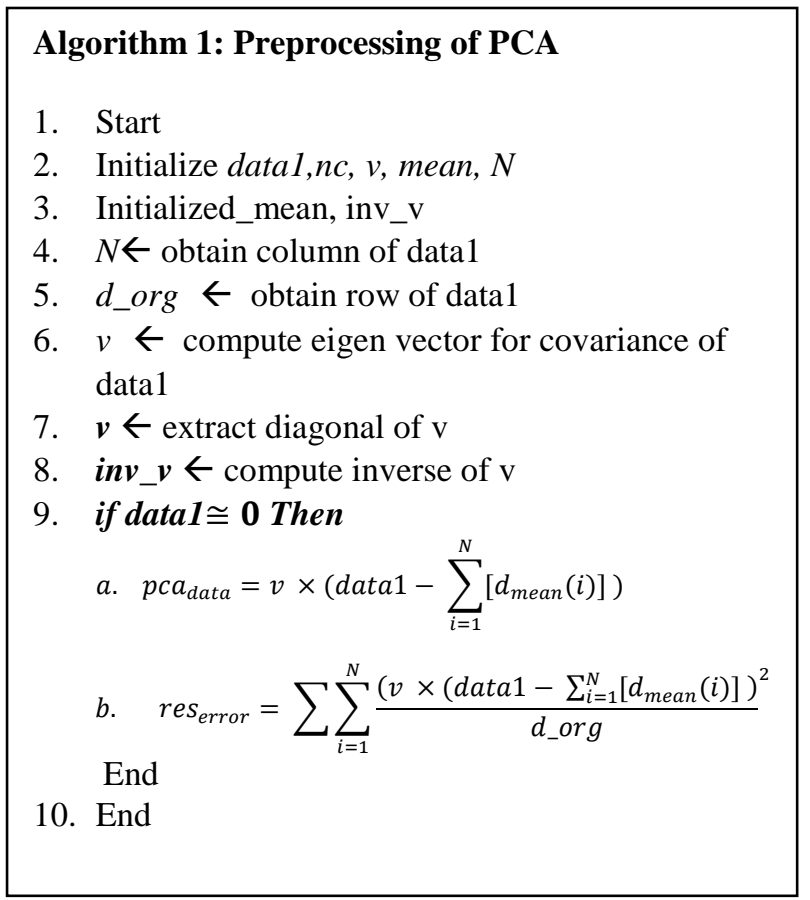

- Conjugate Gradient Descent of non-linearity: In the context of non-linearity in the optimization process, the appropriate method considered for this condition would be conjugate gradient descent algorithm which is a non-linear optimization in nature. For the function of quadratic nature given as $\mathrm{f}(\mathrm{x})$ which is given in (10) below:

$$
f(x)=\|A x-b\|^{2}
$$

Where, $\mathrm{A}, \mathrm{b} \rightarrow$ constraints

At the point when the gradient is 0 , the local minimum is achieved which is given in (11) as shown below:

$$
\nabla_{x} f=2 \cdot A^{T}(A x-b)=0
$$

Where, $\nabla_{x} f \rightarrow$ gradient function with respect to $\mathrm{x}$.

In order to obtain the local minimum, an iterative process called as line search is performed which moves in a particular direction (along the line) in an iterative process until a local minimum is achieved. Here, $\nabla_{x} f$ indicates the direction in which there is an increase in the maximum value. Hence to begin the line search, the initial iterative point begins in the direction opposite (or the steepest descent) to $\nabla_{x} f$. This is represented by the following equation as shown below:

$$
\Delta x_{0}=-\nabla_{x} f\left(x_{0}\right)
$$

To minimize the above mentioned objective function, the linear constraint $\beta_{n}$ is obtained by performing one of the following methods [21], [22]:

$$
\beta_{n}^{F R}=\frac{\Delta x_{n}^{T} \Delta x_{n}}{\Delta x_{n-1}^{T} \Delta x_{n-1}}
$$

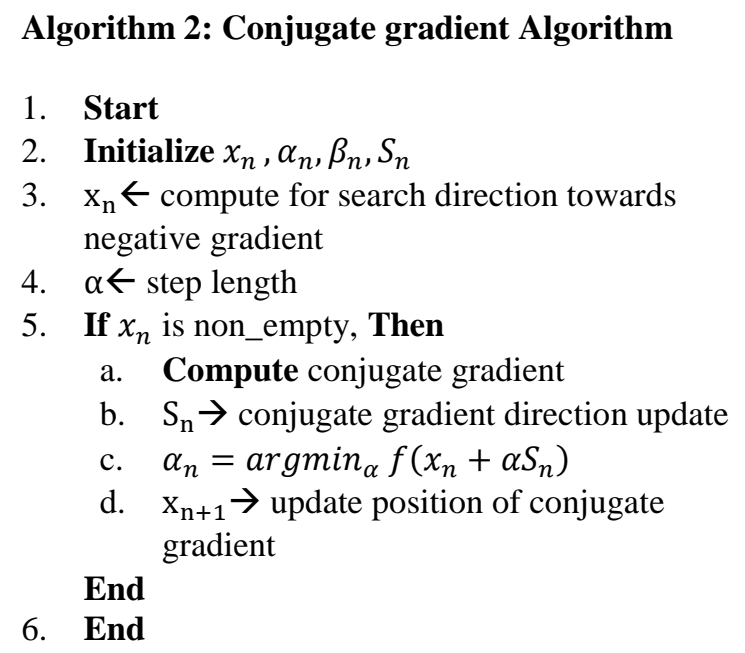

Where, $\beta_{n}^{F R} \rightarrow$ Fletcher- Reeves method for function minimization.

Another method used for function minimization using the conjugate gradient method is the Polak - Ribiere method which is given in (14) as follows:

$$
\beta_{n}^{P R}=\frac{\Delta x_{n}^{T}\left(\Delta x_{n}-\Delta x_{n-1}\right)}{\Delta x_{n-1}^{T} \Delta x_{n-1}}
$$

Where, $\beta_{n}^{P R} \rightarrow$ Polak - Ribiere method for function minimization.

The direction corresponding to the conjugate is updated using the equation given below:

$$
s_{n}=\Delta x_{n}+\beta_{n} s_{n-1}
$$

Where, $s_{n} \rightarrow$ conjugate direction

A line search is performed which computes the local minima along the direction of steepest descent. This is represented in $\mathrm{x}$ as shown below:

$$
\alpha_{n}=\arg \min _{\alpha} f\left(x_{n}+\alpha S_{n}\right)
$$

Where, $\alpha_{n} \rightarrow$ objective function

The position required for the consecutive iteration is updated by the following equation as given below:

$$
x_{n+1}=x_{n}+\alpha_{n} s_{n}
$$

Where, $x_{n+1} \rightarrow$ updated position along the conjugate direction.

The variables used in the development process are represented in the following table: 
TABLE II. PARAMETER DESCRIPTION

\begin{tabular}{|c|c|c|}
\hline Sl.No & Parameter & Description \\
\hline 1. & data1 & Samples considered for training \\
\hline 2. & $\mathrm{x}$ & Samples present in the data1 \\
\hline 3. & $\mathrm{y}$ & Respective weights present in data1 \\
\hline 4. & $\mathrm{v}$ & Eigen vector \\
\hline 5. & $\lambda$ & Eigen value \\
\hline 6. & Q & $\begin{array}{l}\text { Matrix of linear independent Eigen } \\
\text { vectors }\end{array}$ \\
\hline 7. & $\Lambda$ & $\begin{array}{l}\text { Diagonal matrix consisting of Eigen } \\
\text { value }\end{array}$ \\
\hline 8. & $p c a_{\text {data }}$ & Principle components \\
\hline 9. & $d_{\text {mean }}$ & Mean of principle components \\
\hline 10. & res $_{\text {error }}$ & Residual errors \\
\hline 11. & $f(x)$ & Quadratic function \\
\hline 12. & $\mathrm{~A}, \mathrm{~b}$ & constraints \\
\hline 13. & $\nabla_{x} f$ & Gradient function w.r.t $\mathrm{x}$ \\
\hline 14. & $\Delta x_{0}$ & Initial iterative point \\
\hline 15. & $\beta_{n}^{F R}$ & $\begin{array}{l}\text { Function minimization using Fletcher- } \\
\text { Reeves method }\end{array}$ \\
\hline 16. & $\beta_{n}^{P R}$ & $\begin{array}{l}\text { Function minimization using Polak - } \\
\text { Ribiere method }\end{array}$ \\
\hline 17. & $s_{n}$ & Conjugate direction \\
\hline 18. & $\alpha_{n}$ & Objective function \\
\hline 19. & $x_{n+1}$ & $\begin{array}{l}\text { Updated position along conjugate } \\
\text { direction }\end{array}$ \\
\hline
\end{tabular}

\section{RESULTS AND DISCUSSIONS}

In this section the system is analyzed by measuring two parameters which are the mean square error and the regression based correlation coefficient. The input data considered in this experiment is a matrix consisting of 1000 samples with 10 sensors which is given in Table 2 .

- Mean Square Error: The estimation of the prediction error is computed with respect to risk estimation which corresponds to squared loss also sometime called as the quadratic loss. The characteristic of this error is mainly due to the factor of randomness which is present in the estimate. To ensure the quality of the measure with to the estimation the mean square error is used for the same. The MSE is of second order moment which constitutes the variance and its estimation with respect to bias (Fig. 2). The MSE estimation considering the predictor system can be defined as shown in (18) below:

$$
M S E=\frac{1}{n} \sum_{i=1}^{n}\left(\widehat{Y}_{i}-Y_{i}\right)^{2}
$$

Where, $Y_{i} \rightarrow$ vector of observed values

$\hat{Y}_{i} \rightarrow$ Predicted vector

The estimator of the MSE is defined by a variable called $\theta$, which is given as,

$$
\operatorname{MSE}(\hat{\theta})=E\left[(\hat{\theta}-\theta)^{2}\right]
$$

The above defined equation is mainly based on the unknown parameter which in sense is considered to be the property of the estimator of the MSE.
The MSE is considered as the average sum considering the estimators variance and the square bias.

The MSE along with its equivalent variance is given as shown in (20) as follows:

$$
\operatorname{MSE}(\hat{\theta})=\operatorname{Var}(\hat{\theta})+\operatorname{Bias}(\hat{\theta}, \theta)^{2}
$$

- Regression based Correlation coefficient: The coefficient of determination which is considered in this particular metrics is defined as the proportion with respect to variance corresponding to the dependent variable which is obtained from the respective independent variable (Fig. 3).

Considering the mean of the observed data which is represented as $\bar{y}$, its variability is given as,

$$
\bar{y}=\frac{1}{n} \sum_{i=1}^{n} y_{i}
$$

Where, $y_{i} \rightarrow$ modeled vector

The variability of data is computed by using the following methods:

$$
S S_{t o t}=\sum_{i}\left(y_{i}-\bar{y}\right)^{2}
$$

Where, $S S_{\text {reg }} \rightarrow$ sum of squares of total variable

$$
S S_{\text {reg }}=\sum_{i}\left(f_{i}-\bar{y}\right)^{2}
$$

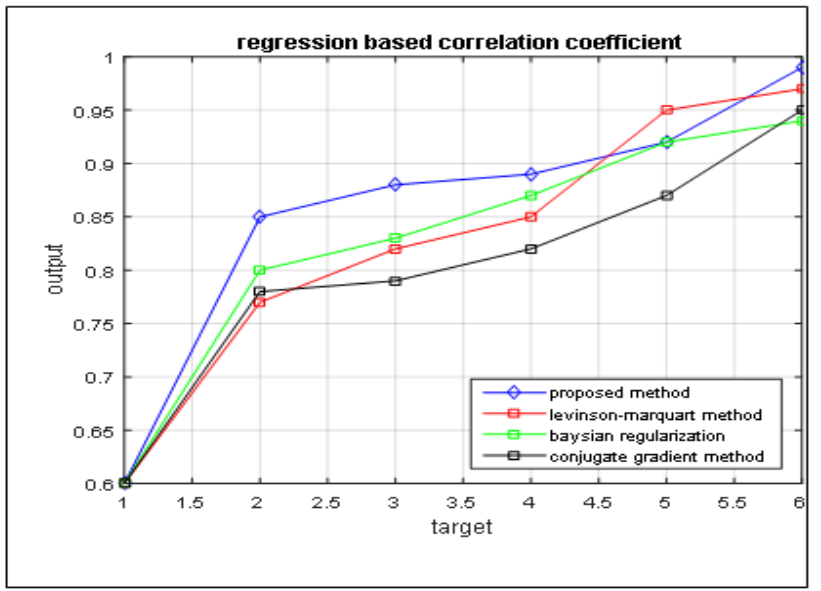

Fig. 2. Comparative analysis for regression based correlation coefficient.

Where, $S S_{\text {reg }} \rightarrow$ regression based sum of squares of total variable

$$
S S_{\text {res }}=\sum_{i}\left(y_{i}-f_{i}\right)^{2}
$$

Where, $S S_{\text {res }} \rightarrow$ residual based sum of squares

The overall general definition of the regression based correlation coefficient is,

$$
R^{2}=1-\frac{S S_{\text {res }}}{S S_{\text {tot }}}
$$

Where, $R^{2} \rightarrow$ coefficient of determination 


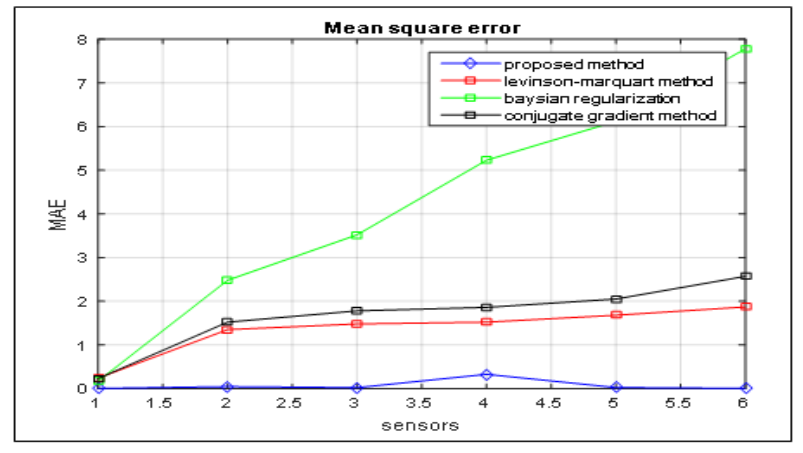

Fig. 3. Comparative analysis with respect to MSE.

The training sample data considered for this experiment is represented in Table 3 as shown below. The network structure of the proposed auto associative neural network considering the bottleneck configuration is represented in Fig. 4.

In Fig. 4 of the AANN architecture, the network configuration considered is here is $\left[\begin{array}{lllll}6 & 12 & 6 & 12 & 6\end{array}\right]$, where a network consisting of 6 neurons is mapped to a network consisting of 12 neurons which is again compressed to a network of 6 neurons which is called as the bottleneck as shown in the above figure. The DE mapping process again consists of a network having 12 neurons which are in turn connected to 6 neurons which are then applied for the process of prediction.

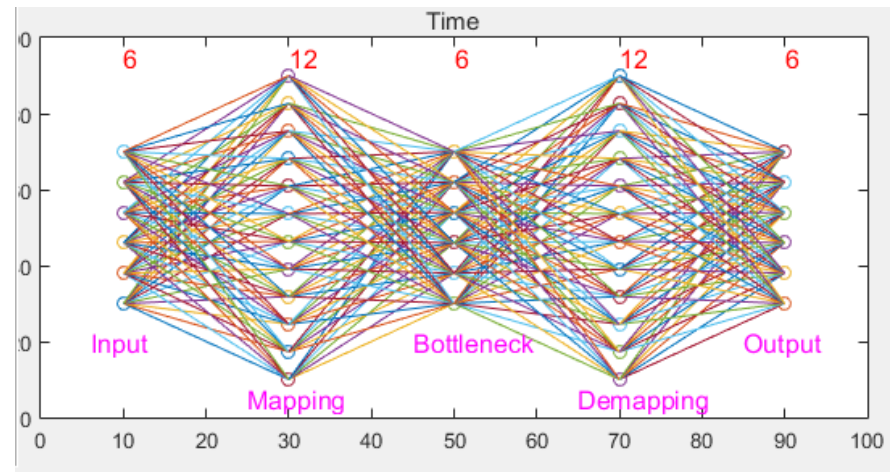

Fig. 4. Network configuration for the NLPCA based AANN system.

TABLE III. DATABASE FOR TRAINING SAMPLES CONSIDERED

\begin{tabular}{|l|c|c|c|c|c|}
\hline $\begin{array}{c}\text { Number of } \\
\text { samples }\end{array}$ & $\mathbf{2 0 0}$ samples & $\mathbf{4 0 0}$ samples & $\mathbf{6 0 0}$ samples & 800 samples & 1000 samples \\
\hline Sensor 3 & 0.003344783 & 0.001562586 & 0.002399367 & 0.008482183 \\
\hline Sensor 4 & 0.046968715 & 0.043513381 & 0.4472326 & 0.046887739 & 0.003442298 \\
\hline Sensor 5 & 0.036573744 & 0.024047742 & 0.021381542 & 0.018151447 & 0.016981448 \\
\hline Sensor 6 & 0.336240087 & 0.326675964 & 0.321492001 & 0.304267503 & 0.305928877 \\
\hline Sensor 8 & 0.023718774 & 0.02296842 & 0.02344671 & 0.02329735 & 0.023237142 \\
\hline Sensor 10 & 0.009956085 & 0.005232597 & 0.004373233 & 0.005903767 & 0.006529151 \\
\hline
\end{tabular}

\section{CONCLUSION}

Experimental results show that the combination of principle component extraction along with conjugate based optimization provides improved results of energy dissipation in WBAN that takes place due to inaccurate prediction, dimensionality reduction, and nonlinearity. A comparative analysis is performed considering the neural network methods such as Baysian regression, Conjugate gradient method, and Levinson-Marquart method. The proposed method shows a significant reduction in the MSE as compared to other methods (Fig. 2). The regression based correlation coefficient has improves in the proposed method as compared to other standard methods (Fig. 3).

\section{REFERENCES}

[1] Li M, Liu J, Ma Z, Yuan C, Yuan B. Throughput optimization with fairness consideration for coexisting WBANs. In2015 IEEE International Conference on Communications (ICC) 2015 Jun 8 (pp. 6418-6423). IEEE.

[2] Kolasa M. Fast and energy efficient learning algorithm for Kohonen Neural Network realized in hardware. acta mechanica et automatica. 2012;6(3):52-7.

[3] Egbogah EE, Fapojuwo AO. Achieving Energy Efficient Transmission in Wireless Body Area Networks for the Physiological Monitoring of Military Soldiers. InMILCOM 2013-2013 IEEE Military Communications Conference 2013 Nov 18 (pp. 1371-1376). IEEE.

[4] Khan JY, Yuce MR, Bulger G, Harding B. Wireless body area network (WBAN) design techniques and performance evaluation. Journal of medical systems. 2012 Jun 1;36(3):1441-57.
[5] Guo-Jian H, Gui-xiong L, Geng-xin C, Tie-qun C. Self-recovery method based on auto-associative neural network for intelligent sensors. InIntelligent Control and Automation (WCICA), 2010 8th World Congress on 2010 Jul 7 (pp. 6918-6922). IEEE.

[6] Gupta AK, Singh YP. Analysis of Bidirectional Associative Memory of Neural Network Method in the String Recognition. InComputational Intelligence and Communication Networks (CICN), 2011 International Conference on 2011 Oct 7 (pp. 172-176). IEEE.

[7] Ang CH, Jin C, Leong PH, van Schaik A. Spiking neural network-based auto-associative memory using FPGA interconnect delays. InFieldProgrammable Technology (FPT), 2011 International Conference on 2011 Dec 12 (pp. 1-4). IEEE.

[8] Lemma TA, Hashim FM. Wavelet analysis and auto-associative neural network based fault detection and diagnosis in an industrial gas turbine. InBusiness Engineering and Industrial Applications Colloquium (BEIAC), 2012 IEEE 2012 Apr 7 (pp. 103-108). IEEE.

[9] Ravi V, Naveen N. Hybrid classifier based on particle swarm optimization trained auto associative neural networks as non-linear principal component analyzer: Application to banking. In2012 12th International Conference on Intelligent Systems Design and Applications (ISDA) 2012 Nov 27 (pp. 77-82). IEEE.

[10] Garimella S, Mallidi SH, Hermansky H. Regularized auto-associative neural networks for speaker verification. IEEE Signal Processing Letters. 2012 Dec;19(12):841-4.

[11] Chakroborty S. Accurate Arrhythmia classification using autoassociative neural network. In2013 35th Annual International Conference of the IEEE Engineering in Medicine and Biology Society (EMBC) 2013 Jul 3 (pp. 4247-4250). IEEE.

[12] Krstulović J, Miranda V, Costa AS, Pereira J. Towards an autoassociative topology state estimator. IEEE transactions on power systems. 2013 Jan 1;28(3):3311-8. 
[13] Zin ZM. Using auto-associative neural networks to compress and visualize multidimensional data. InUbiquitous Robots and Ambient Intelligence (URAI), 2014 11th International Conference on 2014 Nov 12 (pp. 408-412). IEEE.

[14] Ito M, Ohyama W, Wakabayashi T, Kimura F. Rotated face recognition by manifold learning with auto-associative neural network. InFrontiers of Computer Vision (FCV), 2015 21st Korea-Japan Joint Workshop on 2015 Jan 28 (pp. 1-4). IEEE.

[15] Wang C, Yang Y. Robust face recognition from single training image per person via auto-associative memory neural network. InElectrical and Control Engineering (ICECE), 2011 International Conference on 2011 Sep 16 (pp. 4947-4950). IEEE.

[16] Zhang B, Zhou J. Video-based face recognition by Auto-Associative Elman Neural network. InImage and Signal Processing (CISP), 2014 7th International Congress on 2014 Oct 14 (pp. 89-93). IEEE.

[17] Xie Z, Huang G, He J, Zhang Y. A clique-based wban scheduling for mobile wireless body area networks. Procedia Computer Science. 2014 Dec 31;31:1092-101.
[18] Seo S, Bang H, Lee H. Coloring-based scheduling for interactive game application with wireless body area networks. The Journal of Supercomputing. 2016 Jan 1;72(1):185-95.

[19] Islam SR, Kwak KS. A comprehensive study of channel estimation for WBAN-based healthcare systems: feasibility of using multiband UWB. Journal of medical systems. 2012 Jun 1;36(3):1553-67.

[20] Salem O, Guerassimov A, Mehaoua A, Marcus A, Furht B. Anomaly Detection in Medical Wireless Sensor Networks using SVM and Linear Regression Models.

[21] R. Fletcher and C. M. Reeves, "Function minimization by conjugate gradients", Comput. J. 7 (1964), 149-154.

[22] E. Polak and G. Ribi`ere, "Note sur la convergence de directions conjugu'ee", Rev. Francaise Informat Recherche Operationelle, 3e Ann'ee 16 (1969), 35-43. 